\title{
IMAGING
}

\section{Glucose glows in vivo with a new bioluminescent probe}

Maric, T. et al. Nat. Methods 16, 526-532 (2019)

Most organisms wouldn't get far without glucose. But while healthy cells are fueled by the simple sugar, cancerous ones can be too. Too much of the sweet stuff can also lead to metabolic conditions, such as obesity and diabetes. The ability to keep track of glucose metabolism in vivo is thus an important consideration for researchers studying its effects on healthy and not-so-healthy bodies.

Writing in Nature Methods, Tamara Maric and colleagues in Elena Goun's lab at the Swiss Federal Institute of Technology (EPFL) present a bioluminescent glucoseuptake probe dubbed 'BiGluc'. In the proofof-concept paper, the team tested BiGluc in cell lines and in vivo; in one experiment, they show glucose uptake by tumors in a xenograft mouse model. BiGluc's sensitivity was comparable to that of an ${ }^{18} \mathrm{~F}$-FDG-PET tracer, an existing radioactive approach.

A bioluminescent system, found naturally in organisms such as the firefly, was attractive for a few reasons, says Maric. It doesn't require radioactive tracers or expensive PET equipment, and compared to fluorescent reporters there's less background noise to contend with because light is produced from a reaction within the animal rather than from an external source. Attempts by others to use less noisy, near-infrared shifted fluorophores have struggled with glucose because the sugar is just so small compared to the dye that must be attached to it, Maric says; the resulting probes are large and 'sticky', which disrupts uptake into a cell.

Goun's lab spent several years testing and tweaking the biochemistry involved, but they ultimately ended up with a twopart injectable probe that takes advantage of 'click chemistry.' An interested researcher will need: 1) an animal that expresses the luciferase enzyme, which reacts with its substrate luciferin to produce a photon of light; 2) a luciferin that is initially 'caged,' to prevent immediate reactions with the enzyme; and 3) the key that 'clicks' into place, unlocks the cage, and releases the luciferin; for BiGluc, this is a glucose molecule modified with a small azide called GAz4. The caged luciferin is injected intravenously and allowed a few hours to be absorbed into tissue cells; the animal is then given the modified glucose, intraperitoneally or via oral gavage. Each GAz4 key unlocks one cage, resulting in one photon. More glucose taken up by a cell means a brighter signal, says Maric.

The probe functions in real-time and can be administered repeatedly, allowing multiple, non-invasive observations. "I'm very happy with what we have," says Maric, but there's always room to improve-there could still be other chemical tricks to improve BiGluc's sensitivity even more, she says. However, she hopes the probe will be a useful and accessible option for measuring glucose uptake in vivo.

Ellen P. Neff

Published online: 2 July 2019

https://doi.org/10.1038/s41684-019-0356-0
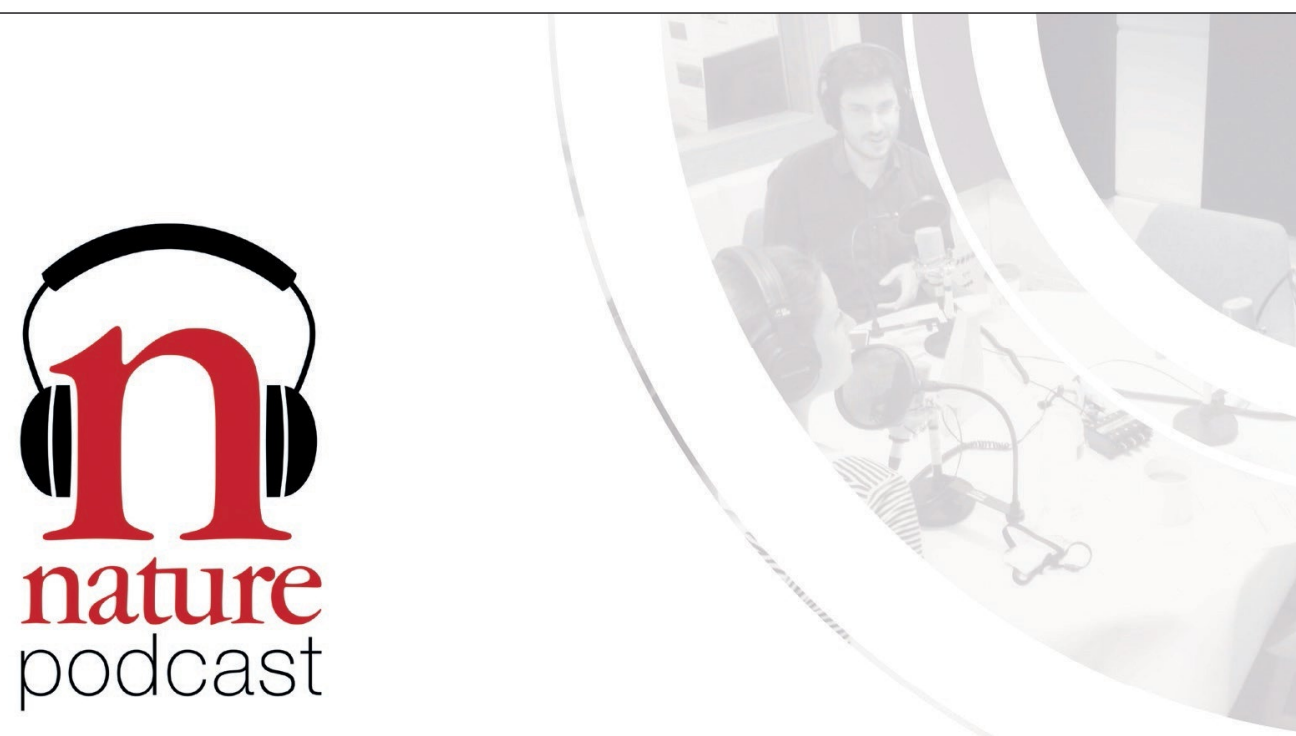

\section{The week's best science,} from the world's \#1 science journal.

NATURE.COM/NATURE/PODCAST 